\title{
Quantitative Phosphotyrosine Proteomics of EphB2 Signaling by Stable Isotope Labeling with Amino Acids in Cell Culture (SILAC)
}

\author{
Guoan Zhang $^{1,2, \#}$, Daniel S. Spellman ${ }^{1,3, \#}$, Edward Y. Skolnik ${ }^{1,2}$, and Thomas A. \\ Neubert ${ }^{1,2^{*}}$ \\ 1 Skirball Institute of Biomolecular Medicine, New York University School of Medicine, New York, New York \\ 10016, USA. \\ 2Department of Pharmacology, New York University School of Medicine, New York, New York 10016, USA. \\ 3New York University and National Institutes of Health Graduate Partnership Program in Structural Biology
}

\section{Abstract}

Eph-related receptor tyrosine kinases (RTK) have been implicated in several biological functions including synaptic plasticity, axon guidance and morphogenesis, yet the details of the signal transduction pathways that produce these specific biological functions after ligand-receptor interaction remain unclear. We used Stable Isotope Labeling by Amino Acids in Cell Culture (SILAC) in combination with LC-MS/MS to characterize cellular signaling following stimulation by ephrinB1-Fc of NG-108 cells that overexpress EphB2 receptors. Because tyrosine phosphorylation functions as a key regulatory event in RTK signaling, we used anti-phosphotyrosine immunoprecipitation (pY IP) of cell lysates to isolate potential participants in the EphB2 pathway. Our SILAC experiments identified 127 unique proteins, 40 of which demonstrated increased abundance in pY IPs from ephrinB1-Fc stimulated cells as compared with unstimulated cells. Six proteins demonstrated decreased abundance, and 81 did not change significantly in relative abundance. Western blotting analysis of five proteins after pY IP verified their SILAC results. Based on previously published work and use of PathwayAssist ${ }^{\mathrm{TM}}$ software, we proposed an interaction network downstream of EphB2 for the proteins with changed ratios.

\author{
Keywords \\ Phosphoproteomics; SILAC; Eph Signaling; Mass Spectrometry
}

\section{Introduction}

The Eph receptors comprise the largest group of receptor tyrosine kinases, with at least 14 members, and are found in a wide variety of cell types in developing and mature tissues. These receptors are activated by another family of cell surface molecules, the ephrins, anchored to the membrane through either a glycosylphosphatidylinositol (GPI) linkage (five ephrin-A's) or through a transmembrane domain (three ephrin-B's), restricting ephrin/Eph receptor signaling to sites of cell-to-cell contact. To date, they have been implicated in a host of biological functions including axon guidance, synaptic plasticity, cell migration, vascular development, tissue-border formation ${ }^{1}$. Recent studies have also suggested an important role for Eph receptors in cancer development and invasiveness ${ }^{2,3}$. Great progress has been made

\footnotetext{
*Correspondence should be addressed to: Thomas A. Neubert, tel.: (212) 263-7265, Fax: (212) 263-8214, E-mail: neubert@saturn.med.nyu.edu.

\#These authors contributed equally to this work
} 
in unraveling the versatile and complex biology of Eph signaling. However, our understanding of the molecular basis for these multiple effects is far from complete, and it is likely that novel Eph-related effectors and signaling mechanisms remain to be discovered.

To begin to address the missing elements of Eph signaling networks, we applied the SILAC (Stable Isotope Labeling by Amino Acids in Cell Culture) approach, which has shown great promise for the simultaneous identification and quantification of complex protein mixtures ${ }^{4-}$ 7. Specifically, SILAC has been demonstrated to be an effective means for characterization of cellular signaling and protein-protein interactions of receptor tyrosine kinases ${ }^{5,8}$. This technology has been successfully applied to the study of EGF and FGF signaling 9,10 . In a typical SILAC experiment, cells representing two different biological conditions are grown in media supplemented with "light" or "heavy" isotope-containing amino acids. Metabolic incorporation of labeled amino acids into all proteins from cells of one population, and subsequent combination of labeled and unlabeled samples in equal ratios, enables quantification of proteins from the two samples based on the intensities of the light and heavy peptides. Within the same mass spectrometric experiment, tandem mass spectrometry (MS/ MS) can be carried out to obtain sequence information for protein identification. Thus, targeted proteomic comparisons can be achieved in a high-throughput way. In the case of ephrin signaling, phosphorylation of tyrosine residues in the kinase and juxtamembrane domains of the Eph receptors regulates their biological and catalytic activities ${ }^{11}$, and tyrosine phosphorylation of downstream effectors plays a key role in ephrin-mediated signaling 12 . Therefore, in our experiments we used SILAC to compare levels of proteins in pY IPs from cells stimulated or unstimulated with ephrinB1-Fc (Figure 1).

NG108 cells, a hybrid mouse neuroblastoma rat glioma line, represents a long standing model of neurons in cell culture ${ }^{13,14}$. Here we use a line of these cells stably expressing the EphB2 receptor in combination with SILAC technology to study protein phosphorylation and complex formation upon application of a soluble ligand. Our results have identified a number of known and novel components of the EphB2 pathway. Based on previous literature reports and in silico analysis, we have proposed a signaling network that includes the proteins that were differentially influenced by ephrin stimulation in our SILAC experiment.

\section{Experimental Section}

\section{Cell culture and metabolic labeling}

Two populations of NG108-15 cells (mouse neuroblastoma $\times$ rat glioma hybrid) stably overexpressing EphB2 ${ }^{12}$ were maintained in lysine and arginine depleted Dulbecco's modified Eagle's medium (DMEM) (Special Media, Philipsburg, NJ) supplemented with 10\% dialyzed fetal bovine serum (Invitrogen Corporation, Carlsbad, CA), HAT (Sigma-Aldrich, St. Louis, MO), 100 units/ml of penicillin / streptomycin, and either normal or ${ }^{13} \mathrm{C}_{6}$ lysine and ${ }^{13} \mathrm{C}_{6}$ arginine (Cambridge Isotope Labs, Andover, MA). Cells were grown for at least 6 doublings to allow full incorporation of labeled amino acids.

\section{Starvation and ephrin stimulation}

After $24 \mathrm{~h}$ of serum starvation, one population was treated for $45 \mathrm{~min}$ with $2 \mu \mathrm{g} / \mathrm{ml}$ ephrinB1Fc (Sigma-Aldrich) that previously had been aggregated using goat anti-human Fc (Jackson Immunoresearch), whereas the other was treated with anti-human $\mathrm{Fc} \mathrm{IgG} \mathrm{only.} \mathrm{The} \mathrm{aggregation}$ was done by incubating ephrinB1-Fc $(250 \mu \mathrm{g} / \mathrm{ml})$ and anti-human $\mathrm{Fc}(65 \mu \mathrm{g} / \mathrm{ml})$ at $4{ }^{\circ} \mathrm{C}$ for 1.5 h. Cells from both conditions were lysed in lysis buffer containing $1 \%$ Triton X-100, 150 $\mathrm{mM} \mathrm{NaCl}, 20 \mathrm{mM}$ Tris, pH8, 0.2mM EDTA, pH8, $2 \mathrm{mM} \mathrm{Na}_{3} \mathrm{VO}_{4}, 2 \mathrm{mM} \mathrm{NaF}$, and protease inhibitors (Complete tablet; Roche, Mannheim, Germany). 


\section{Cell lysis, immunoprecipitation and Western Blot}

Lysates were mixed in a 1:1 ratio (v:v) and pre-cleared by incubating with protein A beads at $4^{\circ} \mathrm{C}$ for $1 \mathrm{~h}$. The lysate was incubated with agarose-conjugated anti-phosphotyrosine antibody PY99 (Santa Cruz) for $4 \mathrm{~h}$, and the beads were washed 4 times with lysis buffer. Precipitated proteins were eluted by boiling in SDS-PAGE sample buffer for $5 \mathrm{~min}$ and separated on a $10 \%$ Tris-HCl gel (Bio-rad). Eluted proteins as well as I.P. supernates were subjected to SDS-PAGE. The gel was stained with Coomassie Blue and the gel lane was cut horizontally into 14 sections. Excised gel bands were cut into small pieces and destained in $25 \mathrm{mM}$ ammonium biocarbonate / $50 \%$ acetonitrile, dehydrated with acetonitrile and dried. The gel pieces were rehydrated with $12.5 \mathrm{ng} / \mu \mathrm{l}$ trypsin solution in $25 \mathrm{mM}$ ammonium bicarbonate and incubated overnight at $37^{\circ}$ C. Peptides were extracted twice with $5 \%$ formic acid / $50 \%$ acetonitrile followed by a final extraction with acetonitrile ${ }^{15}$. Samples were dried by vacuum centrifugation and reconstituted in $6 \mu 10.1 \%$ formic acid/2\% acetonitrile for HPLC sample injection. For western blotting analysis, cell culture, cell treatment and immunoprecipitation were essentially the same way as described above. Precipitated proteins were separated by SDS-PAGE and transferred to PVDF membrane. Membranes were blocked in TBST containing 5\% bovine serum albumin (for anti-phosphotyrosine blots) or skim milk, incubated with the corresponding primary and HRP-conjugated secondary antibodies, and detected with ECL (Santa Cruz Biotechnology, CA, USA).

Anti-EphB2, anti-beta2-chimaerin and anti-Nischarin antibodies were kind gifts from Drs. Matthew B. Dalva, Marcelo G. Kazanietz and Suresh K. Alahari respectively, and were used according to the relevant publications ${ }^{16-18}$. Anti-FAK, anti-Shp2 and PY99-HRP antibodies were purchased (Santa Cruz Biotechnology) and were used as indicated by the manufacturer.

\section{Mass spectrometry, protein identification, and automated quantitation}

The peptide mixtures from tryptic in-gel digestions were analyzed using nanoflow LC/MS/ MS. The peptides were loaded onto a $0.3 \times 1$-mm C18 nano-precolumn (LC Packings, Sunnyvale, CA), then washed 5 min with $2 \%$ ACN in $0.1 \%$ formic acid at a flow rate of $20 \mu \mathrm{l} /$ min. After washing, flow was reversed through the precolumn and the peptides eluted with a gradient of $2-90 \%$ ACN in $0.1 \%$ formic acid. The gradient was delivered over $150 \mathrm{~min}$ by a CapLC (Waters, MA) HPLC system at a flow rate of $200 \mathrm{nl} / \mathrm{min}$, obtained by a 15:1 precolumn flow split, through a $75-\mu \mathrm{m} \times 15-\mathrm{cm}$ fused silica capillary C18 HPLC column (LC Packings PepMap) to a fused silica distal end-coated tip nano-electrospray needle (New Objective, Woburn, MA). The Q-TOF micro (Micromass, Manchester, United Kingdom) data acquisition involved MS survey scans and automatic data-dependent MS/MS acquisitions, which were invoked after selected ions met preset parameters of minimum signal intensity of 12 counts per second, ion charge state $2+, 3+$, or $4+$, and appropriate retention time. Survey scans of $1 \mathrm{~s}$ were followed by CID of the three most intense ions for up to $6 \mathrm{~s}$ each, or until 5,000 total MS/ MS ion counts per precursor peptide were achieved. The raw MS data were subsequently processed using manufacturer-supplied ProteinLynx 3.5 software, with the following settings: Background subtraction of polynomial order 10 below a $10 \%$ curve, 1 smooth with a window of two channels in Savitzky Golay mode, followed by centroid calculation of the top $80 \%$ of peaks based on a minimum peak width of 4 channels at half height. Based on these parameters, pkl files incorporating parent ion mass and retention time as well as peak lists for each corresponding MS/MS spectrum were generated. Mascot software (version 2.0.00, Matrix Science, London, United Kingdom) was used for database search and protein identification using the mouse and rat databases from NCBI (downloaded 10/05/2004) with a minimum parent-ion and fragment-ion mass accuracy of 0.3 Daltons.

For phosphopeptide identification, all phosphopeptide matches from Mascot results were inspected manually. Matches with scores lower than 20 were discarded. We found that all 
phosphopeptides were identified together with their unphosphorylated counterparts with very close retention times in LC. This, together with the similarity between MS/MS spectra of the phosphorylated/unphosphorylated peptides, further improved the confidence for phosphorylation analysis.

Quantification was carried out using the open-source software MSQuant (Peter Mortensen and Matthias Mann, http://msquant.sourceforge.net/). The quantitation data were verified manually for all peptides. As an additional measure to ensure proper quantification, one gel band containing some of the combined cell lysates was subjected to in-gel digestion, LC-MS/MS analysis and the identified proteins were also quantified. The average ratio for all control proteins analyzed was determined and the averaged ratio (1.01:1.00) was used as a correction for ratios of proteins identified from the I.P.

\section{PathwayAssist database analysis}

We used PathwayAssist ${ }^{\mathrm{TM}}$ software (Ariadne Genomics, Rockville, MD, http://www.ariadnegenomics.com) to help construct a model of EphB2 signal transduction for the 46 differentially $\mathrm{pY}$ IPed proteins in our SILAC experiments based on the ResNet (Ariadne Genomics) database. The ResNet(v2.5-Q2) pathway database, which contains more than 500,000 events of regulation, interaction and modification among thousands of proteins, cell processes and small molecules, was modified to include additional information from several databases including PubMed, as well as interactions for EphB2 from the Biomolecular Interaction Database (BIND v3.7, http://www.bind.ca/) and the Database of Interaction Proteins (DIP, http://dip.doe-mbi.ucla.edu/) as of 03/10/05.

\section{Results and Discussion}

\section{SILAC methodology and tyrosine phosphoproteomic analysis of EphB2 signaling}

Complete incorporation of ${ }^{13} \mathrm{C}$ Arg and ${ }^{13} \mathrm{C}$ Lys into NG108-EphB2 cells after 6 cell divisions in isotopically heavy medium was verified by MS analysis of a protein digest (data not shown). Tyrosine phosphorylation has been previously characterized as a key event in both the activation and consequent signal transduction of the ephrin/Eph receptor pathways ${ }^{12}$. As shown in Figure 2, more proteins were tyrosine phosphorylated when cells were stimulated with ephrinB1-Fc[ns3] compared to nonstimulated cells. Thus an anti-phosphotyrosine antibody was used to immunoprecipitate proteins from cell lysates to pull down potential participants in this pathway that were differentially tyrosine phosphorylated upon stimulation as well as proteins that bind to these phosphorylated proteins. Proteins from nonspecific binding were readily determined because their ratios are close to 1 . Because EphB2 is overexpressed in both stimulated and control cell cultures, we expect that proteins that are present as a result of EphB2 overexpression also would be present at ratios close to 1. Figure 3 shows examples of identification and quantification for an up regulated, a down regulated, and a nonspecific binding protein.

To maximize the number of proteins identified, immunoprecipitated proteins were separated by SDS-PAGE and divided into 14 gel slices. Each slice was then digested and analyzed by LC/MS/MS as an individual sample. The SDS-PAGE served as an additional dimension of separation of the intact proteins, which significantly reduced the complexity of samples for LC, allowing detection of low abundance proteins. Although no protein bands except the light and heavy IgG chains from the anti-pY antibody could be observed from the Coomassie stained SDS-PAGE gel, 127 proteins were identified with high confidence, showing the high sensitivity of this method for protein identification. Even in molecular weigh regions where only a weak signal was observed from anti-pY Western blotting, for example, around 75k as shown in Figure 2, a reasonable number of proteins were identified. This indicated that the LC/ 
MS/MS experiments were sensitive enough to allow us to detect low abundance proteins in this complex sample. In cases where a protein was identified in more than one gel slice, protein ratios were consistent suggesting that potential gel mobility shifts caused by phosphorylation were not significant enough to affect our results.

\section{Protein identification and quantification}

Altogether 127 proteins were identified in the SILAC experiment (Supplementary Table 1). Using 1.5 as the threshold for a significant ratio change, 40 proteins were found to be "up regulated" (more abundant in pY IP) upon stimulation and six were "down regulated" (less abundant in pY IP), as shown in Table 1. We chose 1.5 as a conservative cutoff because ratios of proteins found in a mixture of labeled and unlabeled cell lysates, which would expected to be present at a ratio of $1: 1$, were measured with an average standard deviation between peptides within each protein of $11 \%$, and maximum standard deviation of $17 \%$. Most proteins in Table 1 were identified by more than one peptide, and confidence in their correct identifications was high. For those proteins that were identified by a single peptide, the MS/MS spectra were manually inspected to ensure correct identification. For most proteins, the relative standard deviation for quantification between peptides of the same protein was less than $20 \%$. In similar SILAC experiments performed with ten times less material, we obtained similar ratios for each identified protein (Supplementary Table 2 and reference ${ }^{19}$ ). [os6]

To further confirm the SILAC ratios we observed with MS, five proteins in Table 1 were selected for western blot analysis based on availability of antibodies. The selected proteins included the EphB2 receptor and FAK, two known effectors with up and down regulation respectively, and three novel effectors Shp2, beta2-chimaerin and Nischarin. As shown in Figure 4, Western blotting analysis was performed on both the whole cell lysate and pY IPs. For all the selected proteins, their Western blot staining intensities agreed with their SILAC ratios, providing additional evidence for SILAC quantification.

As shown in Table 2, 18 phosphopeptides were found in the SILAC experiment, corresponding to 17 phosphorylation sites, including 12 tyrosine phosphorylation sites. Five phosphorylation sites are reported here for the first time. Figure 5 shows the MS/MS spectra of peptides containing two of the novel sites. One of the peptides shown contains two phosphorylation sites resulting in three different forms of phosphorylation, all of which were characterized unambiguously by their MS/MS spectra[Ds8]. The signaling of Eph receptors, as well as other receptor tyrosine kinases, relies on reversible protein phosphorylation of effector proteins, which generally switches the proteins between activated and deactivated states ${ }^{12}$. It is likely but not necessarily true that the identified phosphorylation sites from proteins that showed significant ratio changes are regulated by the EphB pathway and thus may be important for the signaling cascade. Because in most cases each protein has multiple phosphorylation sites, a change in protein abundance in the pY IP can not be attributed with certainty to phosphorylation or dephosphorylation of any specific site[ss9].

\section{Bioinformatic analysis}

Upon determination of protein identities and calculation of relative ratios between stimulated and unstimulated cells, protein ratio lists were generated and subjected to pathway analysis with the PathwayAssist ${ }^{\mathrm{TM}}$ software tool. This allowed for interpretation of SILAC results in the context of published experimental data about EphB signaling and interactions, as well as available data concerning the additional proteins identified. This included the basic biological functions that have been attributed to them and known relationships between them. We constructed a model pathway by searching our interaction database for direct interactions between proteins we found to be differentially regulated. The validity of the connections was confirmed by inspection of the supporting literature. Results of this analysis show that 23 of 
the proteins found in our experiment to be differentially regulated have been previously characterized as participating in direct interactions with other members of this group. This analysis facilitated the integration of our SILAC data with previous knowledge to obtain a proposed downstream network for EphB2 (Figure 6).

\section{Implications for EphB2 signaling}

Several of the proteins identified in our experiment (Table 1) have been shown to participate in EphB2 signaling in previous reports, such as afadin ${ }^{20}$, SHEP $1^{21}$,Nck, p62 dok , and $\mathrm{RasGAP}^{12}$. Some other proteins have been shown to participate in EphA2 signaling, such as Shp2 and FAK 22,23 , and thus are likely to be related to EphB signaling considering the significant homology between the cytoplasmic domains of EphA2 and EphB2 receptors, and their overlapping cellular functions. This suggests that the strategy we used is capable of finding signaling proteins previously shown to participate in the pathway. Many of the proteins we identified have not been reported previously to associate with Eph receptor signaling, but are involved in functions including cytoskeletal arrangement, cell migration, cell adhesion, endocytosis, and protein and vesicle trafficking. The links between these functions and EphB signaling are discussed below. In addition, Table 1 contains other proteins including those without available functional annotation. These proteins are all candidate effectors for EphB signaling but will not be discussed in detail.

Eph Receptors-Not surprisingly, EphB2 was found to have the highest ratio change after ligand stimulation. To our surprise, however, two other receptors of the Eph family, EphB4 and EphB3, were also found in our experiments and both displayed increased ratios upon stimulation. This indicated that they were activated along with EphB2 either by the ligand directly or they served as substrate for the B2 receptor or downstream kinases ${ }^{24}$. To our knowledge, there has been no previous report of the expression of EphB4 and EphB3 receptors in the NG108 cell line. This finding suggests that in this study, the results observed upon ephrinB1 stimulation of NG108-EphB2 cell line cannot be attributed solely to activation of EphB2, but may also be attributed to stimulation by ephrinB1 of other EphB receptors. It is possible that this observation may also apply to previous investigations of EphB signaling using the same cell line $11,12,20,25-27$.

Cytoskeletal Regulation-A number of proteins found in our experiment to be differentially regulated by ephrinB1 stimulation are involved in cytoskeletal regulation and cell migration/motility. The Nck family of adapter proteins are well established as regulators of actin, and Nck has been shown to form a complex with the activated EphB2 receptor, $\mathrm{p} 62^{d o k}$, and $\mathrm{p} 120 \mathrm{RasGAP} 28,29$. All components of this complex were identified as increasing in ratio after ligand stimulation. Abl2 (also known as Arg), a member of the non-receptor tyrosine kinase family, was also found with an increased ratio after stimulation ${ }^{30-32}$. Recent evidence suggests that the Eph and $\mathrm{Abl}$ families cooperate to regulate actin dynamics in growing axons ${ }^{33}$. Eph receptors have also been demonstrated to regulate actin dynamics through small GTPases of the Rho family (Rho, Rac and Cdc42)(reviewed in ${ }^{34}$ ). The activity of small GTPases is tightly regulated by their association with specific GTPase activating proteins (GAPs) and guanine nucleotide exchange factors (GEFs). Beta2-chimerin and DOCK4, proteins with GAP and GEF activities, respectively, were not previously shown to be involved on Eph signaling. EphB receptors can mediate growth-cone collapse, and inhibition of Rac is essential for this process. Beta2-chimaerin, found to be up regulated in the pY IPs, has demonstrated GAP activity for Rac1 ${ }^{35}$, and alpha2-chimaerin, which is highly homologous to beta2-chimaerin, has been shown to mediate growth-cone collapse induced by semaphorin $3 \mathrm{~A}$ 36. Thus beta2-chimaerin seems to be a promising effector for Rac in the EphB pathway. DOCK4, a specific Rap GTPase, may regulate Rap1 activity in EphB signaling. These proteins, 
along with others shown in Figure 6, suggest several novel downstream targets are responsible for a broad and elaborate regulation of cytoskeletal structures by these receptors.

Junctional Complexes and Cell Adhesion-Several proteins in Table 1 are associated with intercellular junctions. Two tight junction proteins, ZO-1 (Tjp1), a canonical pdz containing protein, and ZO-2 (Tjp2), were both found to be up regulated. They serve as links between integral tight junction proteins and the actin cytoskeleton and as adapters for the recruitment of signaling molecules ${ }^{37}$. ZO-1 has been shown interact with the Ras target afadin, which is tyrosine phosphorylated by the EphB2 receptor ${ }^{38}$. Another cell junction protein found to be more abundant in our pY IPs, delta-catenin, has been shown to be able to regulate Rho GTPases ${ }^{39,40}$. Cortactin, found by us to have a decreased ratio after ephrin stimulation, has been implicated in the formation and/or regulation of cell-cell adhesion and communication, and has been shown to interact with ZO- 1 and afadin ${ }^{41}$. Taken together, the results imply these proteins play roles in modulating cell adhesion and actin rearrangement following EphB activation.

Shp2 has been implicated in the regulation of cell spreading, migration, and focal adhesion $^{42,43}$. Eph receptors are known to be capable of modulating cell adhesion and multiple hypotheses on how Shp2 may be involved have been proposed. Miao et al. showed that the protein tyrosine phosphatase Shp2 is recruited to EphA2 receptor upon receptor activation, and subsequently dephosphorylates FAK, leading to negative regulation of cell adhesion ${ }^{23}$. However, in another report, Carter et al. reported the opposite observation that FAK and p-130cas are phosphorylated, rather than dephosphorylated, upon EphA2 activation ${ }^{22}$. There are no reports of similar experiments using EphB receptors. Interestingly, in our SILAC experiment, Shp2, FAK and p-130cas were all identified. Shp2 was enriched by pY IP, while FAK and p-130cas displayed decreased ratios (Table 1), suggesting the cell adhesion regulation upon EphB activation in the EphB2-NG 108 cell model we used is likely to follow the pathway described by Miao et al.

Endocytosis and Protein trafficking-Many activated RTKs are attenuated by internalization and transported through endosomes to multivesicular bodies before recycling to the membrane or delivery to the lysosome for degradation. Therefore, it is not surprising that ubiquitin, E3 ubiquitin-protein ligase, and ubiquitin specific protease 15 showed increased ratios after ligand stimulation, though the degradation process for Eph receptors specifically has not been described in the literature. The fact that ubiquitin was found in the same gel band as EphB2 indicates that the increased ratio of ubiquitin should be mainly attributed to ubiquitination of EphB2 receptor, as EphB2 receptor is the dominant protein in that band. Consistent with this observation, several proteins associated with endocytosis and trafficking also exhibited increased ratio after stimulation, such as signal transducing adaptor molecule (STAM1), HGF-regulated tyrosine kinase substrate (Hrs), Rab 7, Trafficking protein particle complex 5, similar to Epilepsy holoprosencephaly candidate-1 protein (EHOC-1) and Alix ${ }^{44-46}$. This result further supports the previous studies that EphB activation can induce rapid endocytosis, which is critical for separating ligand expressing cells from receptor expressing cells after repulsive signaling by the membrane-associated ephrins and Ephs 47 , 48 . The above mentioned proteins suggest a number of specific targets by which EphB signaling could modulate multiple components of endocytic pathways.

\section{Conclusions}

Our SILAC study confirmed the involvement of many proteins previously involved in EphB signaling. In addition, many proteins not previously known to be involved in EphB signaling also were identified. Consistent with the emerging view of a complex signaling network downstream of EphB receptors, many of these proteins are involved in various cellular 
functions including cytoskeleton arrangement, trafficking, cell adhesion/migration, endocytosis, and cell proliferation. By searching the literature describing known interactions, we were able to describe a potential EphB2 signaling network based on our results. Our experiments have revealed candidate proteins for future research into these Eph signaling pathways.

\section{Supplementary Material}

Refer to Web version on PubMed Central for supplementary material.

\section{Acknowledgements}

This work was supported by NIH grants R21 NS44184 and S10 RR017990 to TAN and NIH IRTA Fellowship to DSS. We thank Matthew B. Dalva for the EphB2 antibody, Marcelo G. Kazanietz for the beta2-chimaerin antibody, Suresh K. Alahari for the Nischarin antibody, Dimitar Nikolov for ephrinB1-Fc, Matthias Mann, Peter Mortensen and Joost Gouw for assistance in adapting MSQuant for our data, Jiri Zavadil and the NYU Cancer Institute Genomics Facility for providing access to PathwayAssist and Tony Pawson for the NG108-EphB2 cell line. We thank Dr. Vivekananda Shetty for expert help with Q-TOF LC-MS/MS.

\section{References}

1. Flanagan JG, Vanderhaeghen P. Annu Rev Neurosci 1998;21:309-345. [PubMed: 9530499]

2. Duxbury MS, Ito H, Zinner MJ, Ashley SW, Whang EE. Biochem Biophys Res Commun 2004;320:1096-1102. [PubMed: 15249202]

3. Fox BP, Kandpal RP. Biochem Biophys Res Commun 2004;318:882-892. [PubMed: 15147954]

4. Ong SE, Foster LJ, Mann M. Methods 2003;29:124-130. [PubMed: 12606218]

5. Blagoev B, Kratchmarova I, Ong SE, Nielsen M, Foster LJ, Mann M. Nat Biotechnol 2003;21:315318. [PubMed: 12577067]

6. Oda Y, Huang K, Cross FR, Cowburn D, Chait BT. Proc Natl Acad Sci U S A 1999;96:6591-6596. [PubMed: 10359756]

7. Ong SE, Blagoev B, Kratchmarova I, Kristensen DB, Steen H, Pandey A, Mann M. Mol Cell Proteomics 2002;1:376-386. [PubMed: 12118079]

8. Ong SE, Kratchmarova I, Mann M. J Proteome Res 2003;2:173-181. [PubMed: 12716131]

9. Hinsby AM, Olsen JV, Mann M. J Biol Chem 2004;279:46438-46447. [PubMed: 15316024]

10. Blagoev B, Ong SE, Kratchmarova I, Mann M. Nat Biotechnol 2004;22:1139-1145. [PubMed: 15314609]

11. Binns KL, Taylor PP, Sicheri F, Pawson T, Holland SJ. Mol Cell Biol 2000;20:4791-4805. [PubMed: 10848605]

12. Holland SJ, Gale NW, Gish GD, Roth RA, Songyang Z, Cantley LC, Henkemeyer M, Yancopoulos GD, Pawson T. Embo J 1997;16:3877-3888. [PubMed: 9233798]

13. Hamprecht B, Glaser T, Reiser G, Bayer E, Propst F. Methods Enzymol 1985;109:316-341. [PubMed: 2985920]

14. Hamprecht B. Int Rev Cytol 1977;49:99-170. [PubMed: 16829]

15. Shevchenko A, Wilm M, Vorm O, Mann M. Anal Chem 1996;68:850-858. [PubMed: 8779443]

16. Dalva MB, Takasu MA, Lin MZ, Shamah SM, Hu L, Gale NW, Greenberg ME. Cell 2000;103:945956. [PubMed: 11136979]

17. Caloca MJ, Wang H, Delemos A, Wang S, Kazanietz MG. J Biol Chem 2001;276:18303-18312. [PubMed: 11278894]

18. Alahari SK, Reddig PJ, Juliano RL. Embo J 2004;23:2777-2788. [PubMed: 15229651]

19. Zhang G, Neubert TA. Mol Cell Proteomics. 2005

20. Hock B, Bohme B, Karn T, Yamamoto T, Kaibuchi K, Holtrich U, Holland S, Pawson T, RubsamenWaigmann H, Strebhardt K. Proc Natl Acad Sci U S A 1998;95:9779-9784. [PubMed: 9707552]

21. Dodelet VC, Pazzagli C, Zisch AH, Hauser CA, Pasquale EB. J Biol Chem 1999;274:31941-31946. [PubMed: 10542222] 
22. Carter N, Nakamoto T, Hirai H, Hunter T. Nat Cell Biol 2002;4:565-573. [PubMed: 12134157]

23. Miao H, Burnett E, Kinch M, Simon E, Wang B. Nat Cell Biol 2000;2:62-69. [PubMed: 10655584]

24. Freywald A, Sharfe N, Roifman CM. J Biol Chem 2002;277:3823-3828. [PubMed: 11713248]

25. Tong J, Elowe S, Nash P, Pawson T. J Biol Chem 2003;278:6111-6119. [PubMed: 12486127]

26. Elowe S, Holland SJ, Kulkarni S, Pawson T. Mol Cell Biol 2001;21:7429-7441. [PubMed: 11585923]

27. Kong H, Boulter J, Weber JL, Lai C, Chao MV. J Neurosci 2001;21:176-185. [PubMed: 11150334]

28. Buday L, Wunderlich L, Tamas P. Cell Signal 2002;14:723-731. [PubMed: 12034353]

29. Becker E, Huynh-Do U, Holland S, Pawson T, Daniel TO, Skolnik EY. Mol Cell Biol 2000;20:15371545. [PubMed: 10669731]

30. Pendergast AM. Adv Cancer Res 2002;85:51-100. [PubMed: 12374288]

31. Van Etten RA, Jackson PK, Baltimore D, Sanders MC, Matsudaira PT, Janmey PA. J Cell Biol 1994;124:325-340. [PubMed: 8294516]

32. Wang Y, Miller AL, Mooseker MS, Koleske AJ. Proc Natl Acad Sci U S A 2001;98:14865-14870. [PubMed: 11752434]

33. Yu HH, Zisch AH, Dodelet VC, Pasquale EB. Oncogene 2001;20:3995-4006. [PubMed: 11494128]

34. Noren NK, Pasquale EB. Cell Signal 2004;16:655-666. [PubMed: 15093606]

35. Caloca MJ, Wang H, Kazanietz MG. Biochem J 2003;375:313-321. [PubMed: 12877655]

36. Brown M, Jacobs T, Eickholt B, Ferrari G, Teo M, Monfries C, Qi RZ, Leung T, Lim L, Hall C. J Neurosci 2004;24:8994-9004. [PubMed: 15483118]

37. Matter K, Balda MS. Nat Rev Mol Cell Biol 2003;4:225-236. [PubMed: 12612641]

38. Yokoyama S, Tachibana K, Nakanishi H, Yamamoto Y, Irie K, Mandai K, Nagafuchi A, Monden M, Takai Y. Mol Biol Cell 2001;12:1595-1609. [PubMed: 11408571]

39. Franz CM, Ridley AJ. J Biol Chem 2004;279:6588-6594. [PubMed: 14660598]

40. Anastasiadis PZ, Reynolds AB. Curr Opin Cell Biol 2001;13:604-610. [PubMed: 11544030]

41. Katsube T, Takahisa M, Ueda R, Hashimoto N, Kobayashi M, Togashi S. J Biol Chem 1998;273:29672-29677. [PubMed: 9792678]

42. Saxton TM, Pawson T. Proc Natl Acad Sci U S A 1999;96:3790-3795. [PubMed: 10097116]

43. Yu DH, Qu CK, Henegariu O, Lu X, Feng GS. J Biol Chem 1998;273:21125-21131. [PubMed: 9694867]

44. Bache KG, Raiborg C, Mehlum A, Stenmark H. J Biol Chem 2003;278:12513-12521. [PubMed: 12551915]

45. Mizuno E, Kawahata K, Kato M, Kitamura N, Komada M. Mol Biol Cell 2003;14:3675-3689. [PubMed: 12972556]

46. Katoh K, Shibata H, Suzuki H, Nara A, Ishidoh K, Kominami E, Yoshimori T, Maki M. J Biol Chem 2003;278:39104-39113. [PubMed: 12860994]

47. Zimmer M, Palmer A, Kohler J, Klein R. Nat Cell Biol 2003;5:869-878. [PubMed: 12973358]

48. Marston DJ, Dickinson S, Nobes CD. Nat Cell Biol 2003;5:879-888. [PubMed: 12973357] 


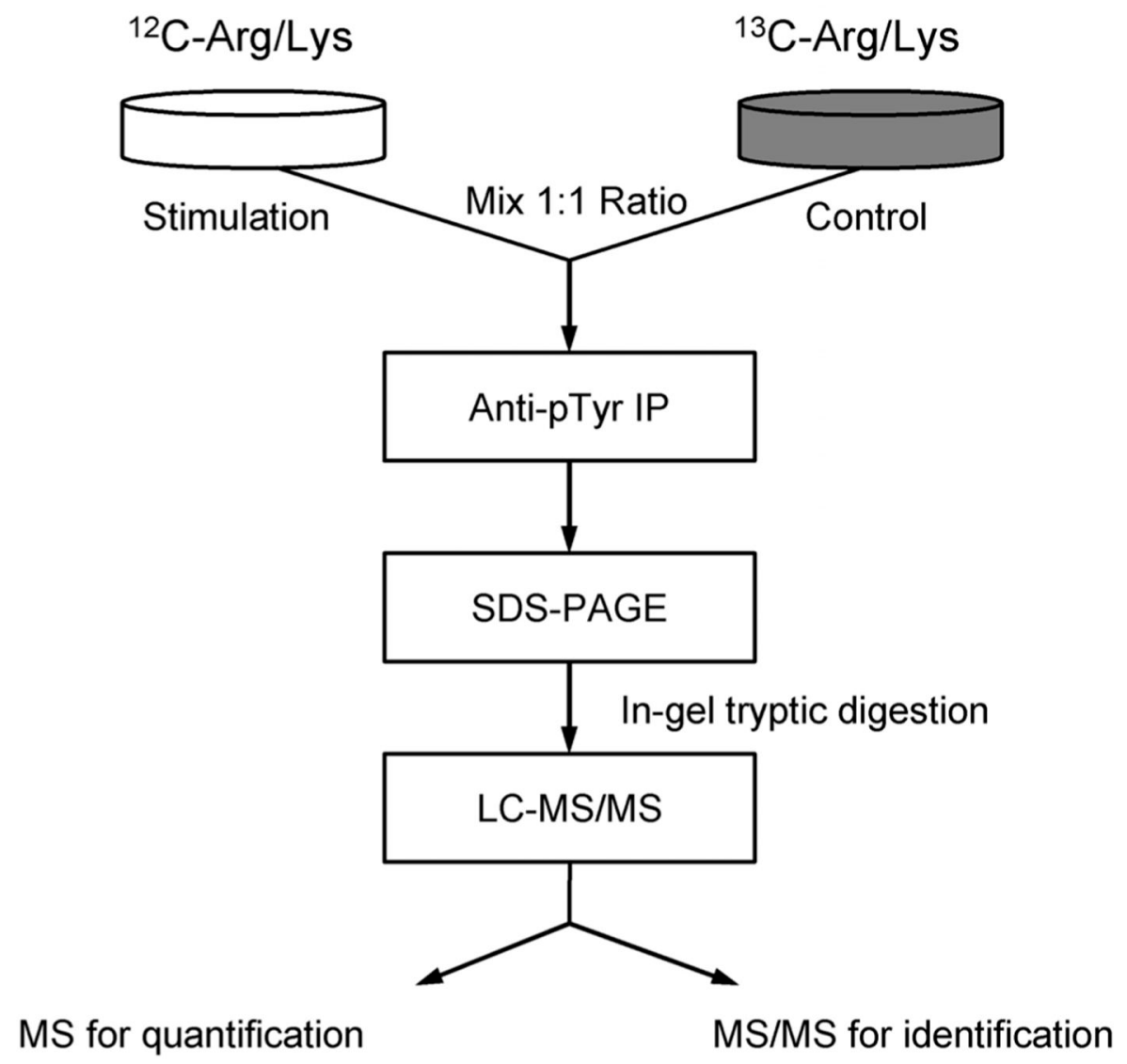

Figure 1.

Strategy to study EphB signaling with SILAC. One cell population is grown in medium containing normal Arg and Lys, whereas another is grown in medium containing heavy Arg and Lys. One of the cell populations was stimulated with aggregated ephrinB1, while the other population was left untreated as a control. The cell lysates from stimulated and control cells were combined at a 1:1 ratio for anti-pY IP. The precipitated proteins were separated by SDSPAGE. The gel lane was cut into 14 bands, digested in-gel with trypsin and analyzed by LCMS/MS for protein identification and quantification. 


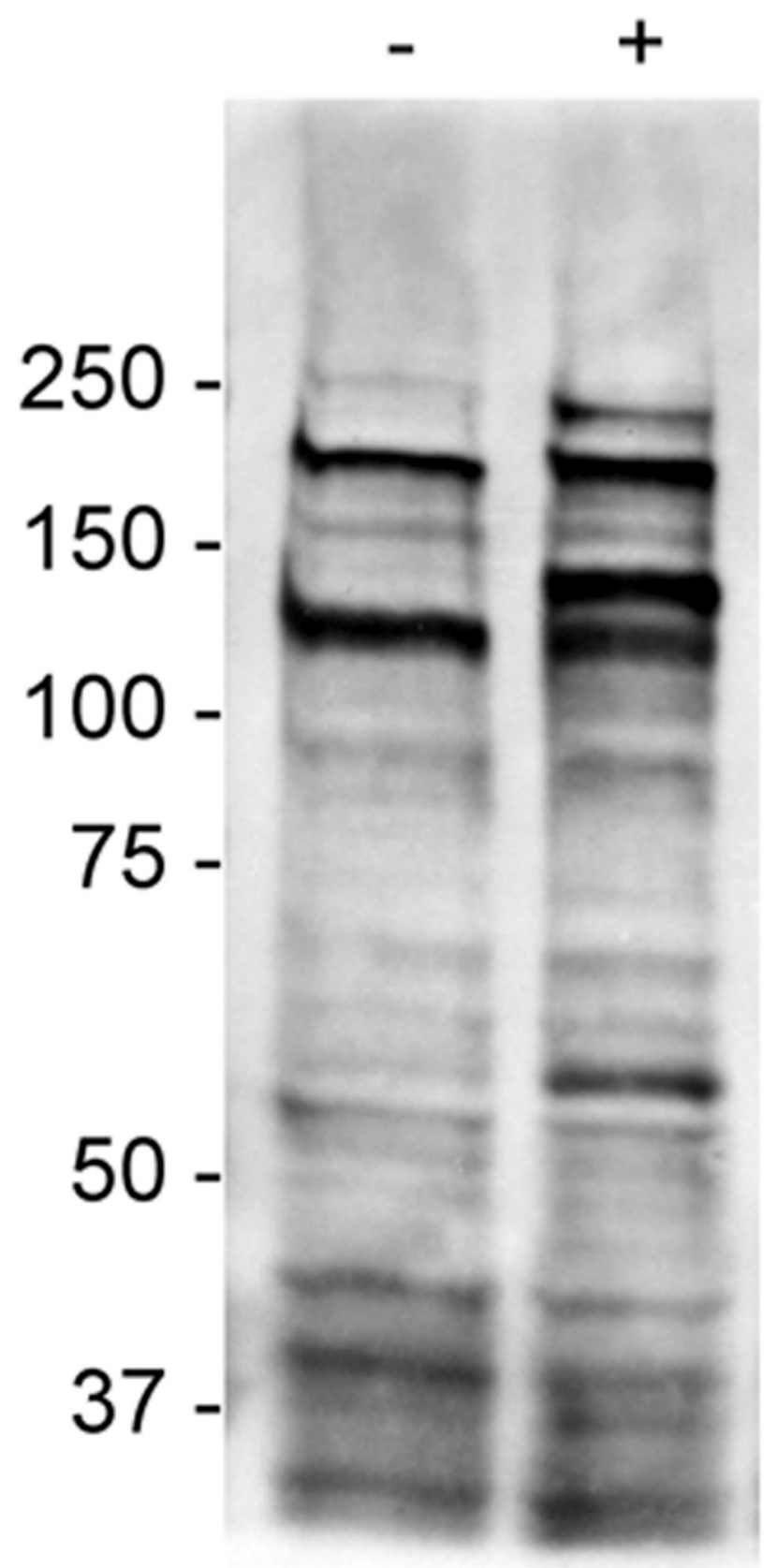

Figure 2.

Protein phosphorylation after ephrinB1-Fc stimulation detected by anti-pY Western blotting. NG108-EphB2 cells were treated with either anti-Fc IgG or ephrinB1-Fc as indicated in the experimental section. Total cell lysates were probed with PY99-HRP[Ds4]. 
EphB2

FLEDDTSDPTYTSALGGK, ratio $=14.3$

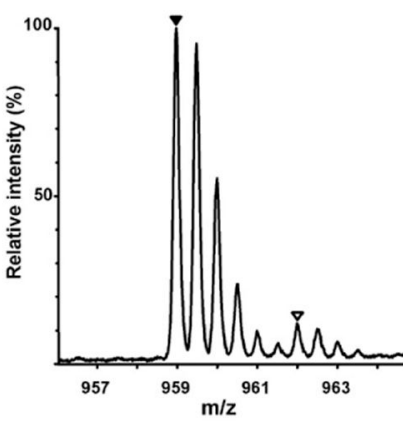

FAK

VFHYFESSSEPTTWASIIR, ratio $=0.67$

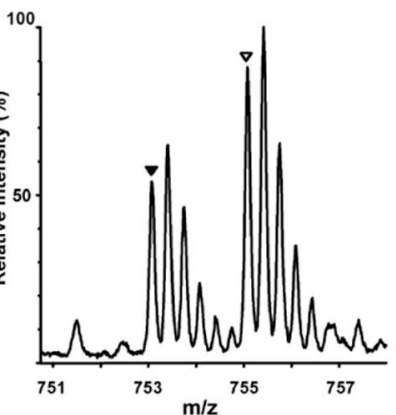

Myosin

QLLQANPILEAFGNAK, ratio $=1.0$

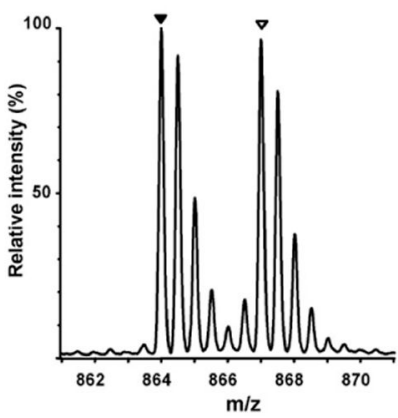

Figure 3.

Quantification of SILAC proteins. The lower-mass peak clusters are from nonlabeled peptides from the stimulated cells while the higher-mass peak clusters are from heavy Arg/Lys labeled peptides from the control cells. Ratios were determined by comparing the heights of the peaks from nonlabeled and labeled peptides. The left panel shows a SILAC peptide doublet from the EphB2 receptor, which was highly enriched in the anti-pY IP after ephrinB1 stimulation. The middle panel shows a peptide doublet from FAK, which was less abundant in the anti-pY IP after ephrinB1 stimulation. The right panel shows a peptide pair from myosin with a ratio of $1: 1$, indicating this protein does not participate in ephrin signaling. 


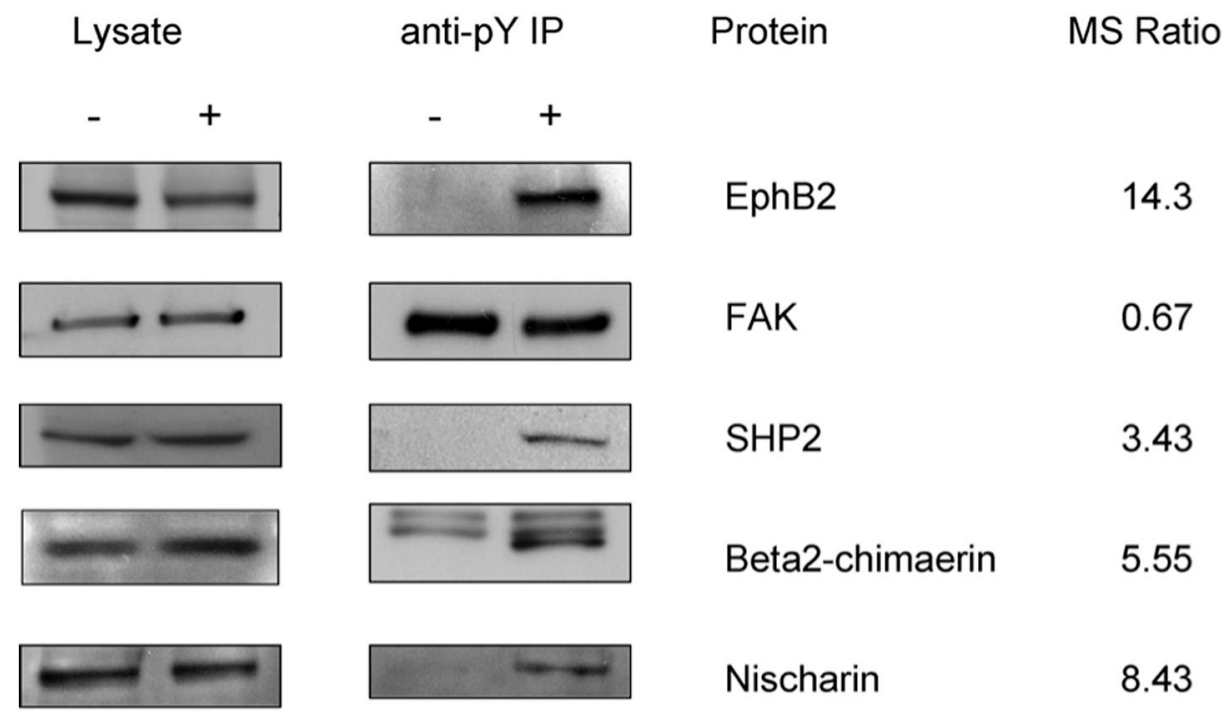

Figure 4.

Western blotting analysis of selected EphB2 signaling proteins. Cells were cultured and stimulated with ephrinB1 in the same way as in the SILAC experiments. Anti-pY IP samples were probed with the indicated antibodies. The total cell lysates were also probed as controls. The two upper bands present in the two rightmost lanes of the beta2-chimaerin Western blot were due to nonspecific staining with secondary antibodies (data not shown). 
(A)

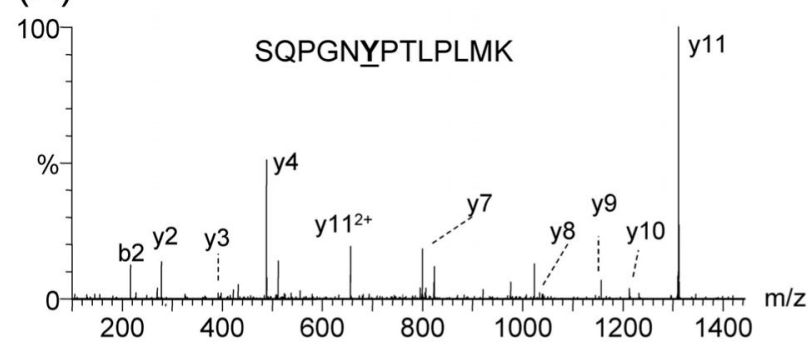

(C)

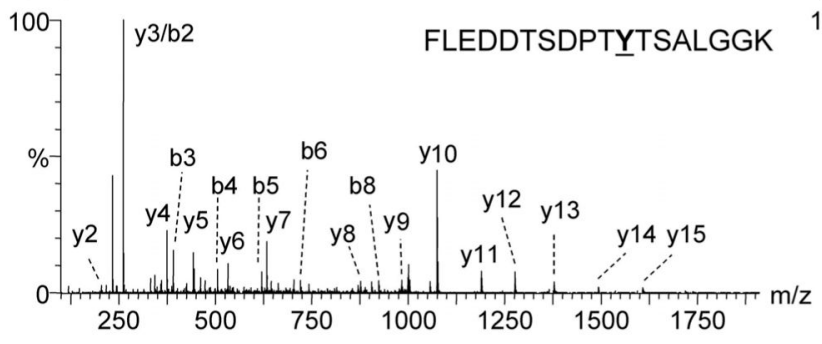

(B)

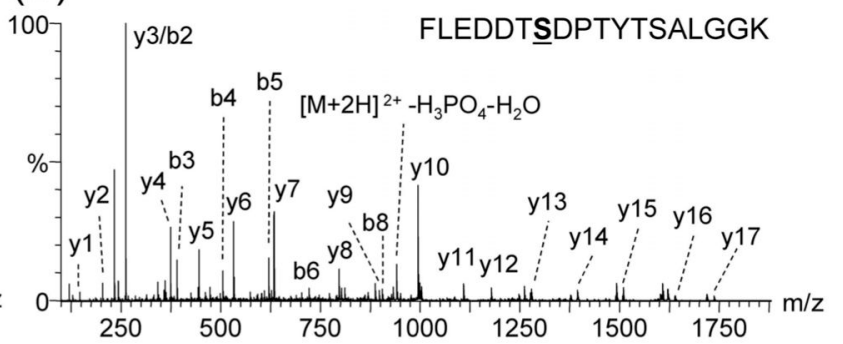

(D)

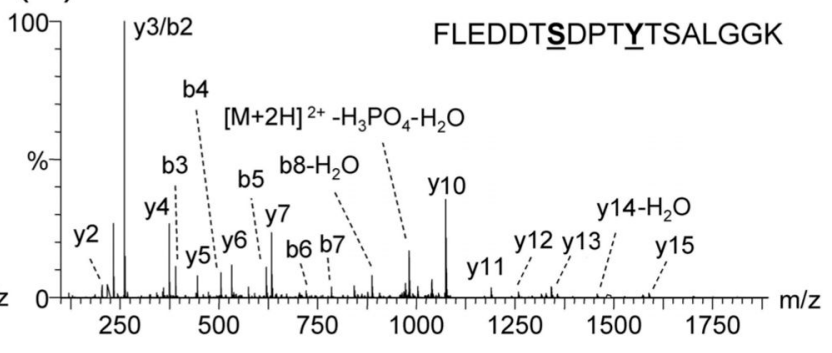

Figure 5.

MS/MS spectra of phosphopeptide SQPGNYPTLPLMK from SASH1 (panel A) and

FLEDDTSDPTYTSALGGK from EphB2 with Ser753 (panel B), Tyr757 (panel C) and both sites (panel D) phosphorylated. Phosphorylated residues are underlined in the spectra. The $b$ and y ions represent the corresponding fragment ions after loss of $\mathrm{H}_{3} \mathrm{PO}_{4}$ (for every $\mathrm{pS}$ site) and $\mathrm{HPO}_{3}$ (for every $\mathrm{pY}$ site). M stands for the precursor peptide. 


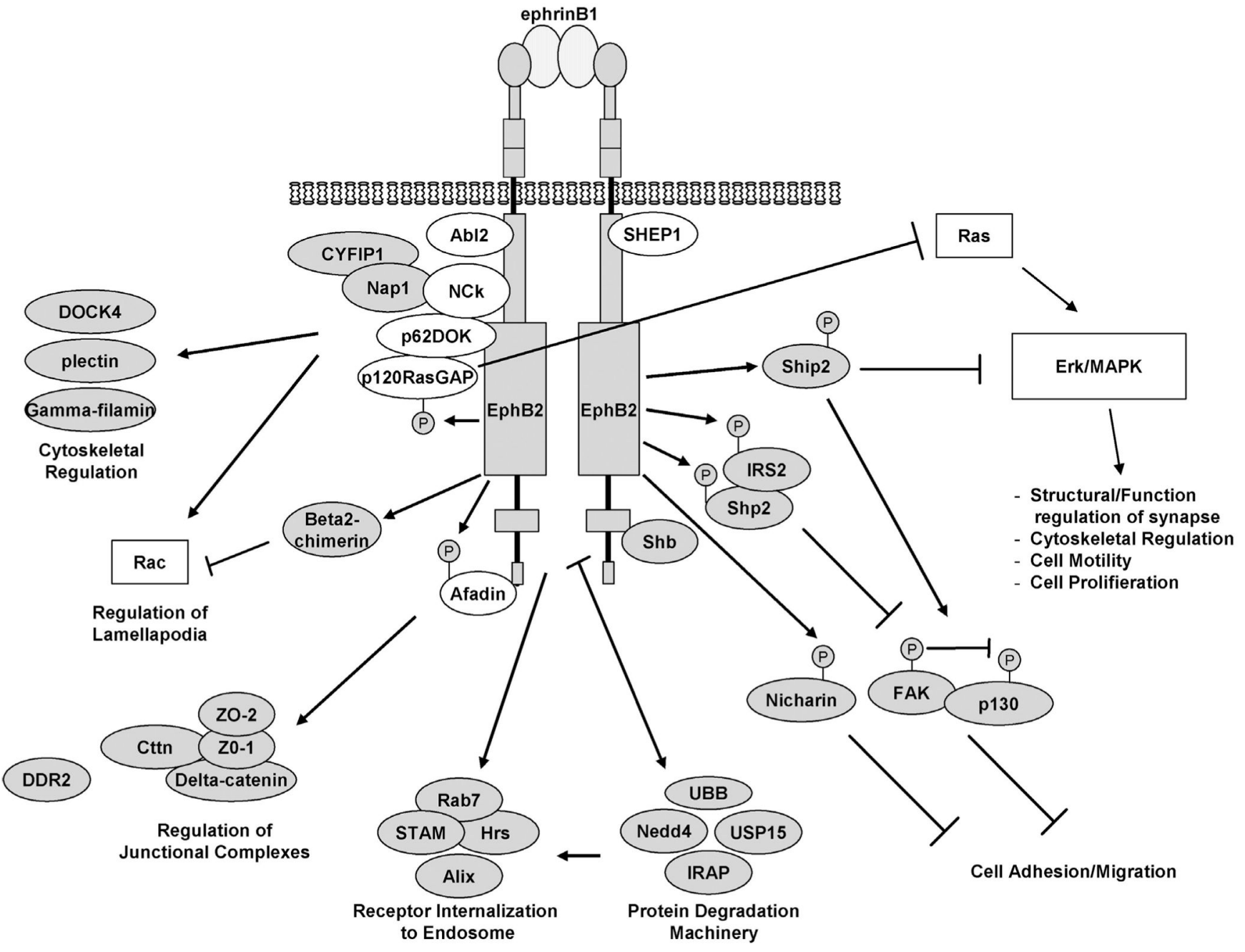

Figure 6.

Proposed EphB2 signaling network. The proposed pathway reflects SILAC data,

PathwayAssist ${ }^{\mathrm{TM}}$ data, and interactions and protein functions found in the primary literature. Proteins in white ovals indicate previously established EphB2 interactions. Arrows indicate positive regulation or activation and blunt ended lines indicate inhibition. Proteins in boxes (Rac, Ras, and Erk) indicate known effectors not found in our experiments. 
Table 1

Ratios of protein abundance in anti-phosphotyrosine immunoprecipitates from ephrinB1-stimulated and unstimulated NG108-EphB2 cells.

\begin{tabular}{|c|c|c|c|c|}
\hline gi No. & Protein Name & Ratio & S.D. & $\begin{array}{c}\text { No. of Unique Peptides } \\
\text { ID'ed }\end{array}$ \\
\hline 38605719 & EphB2 & 14.33 & 4.99 & 19 \\
\hline 31981796 & docking protein $1\left(\mathrm{p} 62^{\mathrm{dok}} / \mathrm{DOK} 1\right)$ & 13.46 & - & 1 \\
\hline 34858379 & similar to adiponectin receptor 1 & 13.32 & - & 1 \\
\hline 92022 & Rab7 & 12.34 & 1.09 & 9 \\
\hline 6978469 & Afadin (AF6) & 9.42 & 2.38 & 29 \\
\hline 12313873 & Nischarin & 8.43 & 1.62 & 3 \\
\hline 31541896 & Putative adapter and scaffold protein 1 (SASH 1) & 6.66 & 3.32 & 11 \\
\hline 1708165 & EphB3 & 6.32 & 1.82 & 2 \\
\hline 631806 & Beta2-chimaerin & 5.55 & 0.10 & 3 \\
\hline 1708335 & EphB4 & 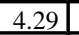 & 2.82 & 7 \\
\hline 4249651 & Nck & 4.11 & 0.17 & 5 \\
\hline 6755668 & Signal transducing adaptor molecule (STAM1) & 4.08 & 0.93 & 6 \\
\hline 21703900 & p120-RasGAP (RAS p21 protein activator 1 ) & 3.99 & 0.82 & 15 \\
\hline 34852356 & Similar to Epilepsy holoprosencephaly candidate-1 protein (EHOC-1) & 3.80 & - & 2 \\
\hline 30840992 & RIKEN cDNA 1810044A24 & 3.77 & 0.35 & 2 \\
\hline 545100 & $\mathrm{Shb}=\mathrm{Src}$ homology 2 protein $(\mathrm{Shb})$ & 3.71 & - & 1 \\
\hline 28380066 & Protein KIAA1688 homolog & 3.65 & 1.87 & 7 \\
\hline 25059002 & Tight junction protein 2 (ZO-2/Tip2) & 3.44 & - & 3 \\
\hline 464495 & Protein-tyrosine phosphatase (Shp2) & 3.43 & 0.88 & 3 \\
\hline 6678355 & Tight junction protein 1 (ZO-1/Tip1) & 3.21 & 0.37 & 8 \\
\hline 33859566 & Inositol polyphosphate phosphatase-like 1(SHIP2) & 3.10 & 0.33 & 10 \\
\hline 6225951 & Rho-interacting protein 3 (RIP3) & 3.10 & - & 2 \\
\hline 32451614 & delta-catenin/Catns protein & 2.96 & - & 1 \\
\hline 1089781 & HGF-regulated tyrosine kinase substrate (Hrs) & 2.95 & 0.71 & 4 \\
\hline 14195008 & Plectin 1 (PLTN) & 2.95 & 1.30 & 2 \\
\hline 29165850 & Trafficking protein particle complex 5 & 2.83 & - & 1 \\
\hline 34871864 & Similar to High-glucose-regulated protein 8 (NY-REN-2 antigen) & 2.76 & 0.35 & 2 \\
\hline 3024044 & Insulin Receptor Substrate-2 (IRS2) & 2.72 & 0.14 & 4 \\
\hline 9506475 & Cell division cycle 2 homolog $\mathrm{A}$ (Cdc2a) & 2.41 & 0.29 & 7 \\
\hline 27370240 & Leucyl/cystinyl aminopeptidase (IRAP) & 2.16 & 0.76 & 2 \\
\hline 32469672 & Dedicator of cytokinesis protein 4 (DOCK4) & 2.10 & - & 2 \\
\hline 91870 & Polyubiquitin & 2.08 & - & 3 \\
\hline 1374782 & E3 ubiquitin-protein ligase Nedd-4 & 1.98 & 0.33 & 3 \\
\hline 21489969 & Ubiquitin specific protease 15 & 1.96 & 0.04 & 2 \\
\hline 51705305 & $\mathrm{v}$-abl Abelson murine leukemia viral oncogene $2(\mathrm{Abl} / \mathrm{Arg})$ & 1.90 & 0.13 & 3 \\
\hline 34855059 & Similar to gamma-filamin & 1.87 & 0.29 & 4 \\
\hline 7304993 & drebrin-like & 1.61 & - & 1 \\
\hline 1708980 & Nck-associated protein 1 (Nap1) & 1.55 & - & 1 \\
\hline 7242205 & Cytoplasmic FMR1 interacting protein 1(CYFRP1/Sra-1/Shyc) & 1.51 & 0.20 & 10 \\
\hline 6755002 & Programmed cell death 6 interacting protein & 1.51 & 0.24 & 3 \\
\hline 26348235 & FAK (PTK2) & 0.67 & 0.05 & 46 \\
\hline 6679207 & Contactin 3 & 0.63 & - & 4 \\
\hline 15030315 & Cortactin $(\mathrm{Cttn})$ & 0.61 & 0.20 & 7 \\
\hline 71897 & GTP-binding regulatory protein $\mathrm{Gi}$ alpha- 2 chain & 0.59 & 0.06 & 2 \\
\hline 6906729 & Cas and HEF1 associated signal transducer (SHEP1) & 0.56 & 0.03 & 5 \\
\hline 2497565 & Discoidin domain receptor 2 (DDR2) & 0.56 & - & 1 \\
\hline
\end{tabular}

-: unable to calculate S.D. because only one peptide is available for quantification 
Table 2

Characterization of phosphopeptides from anti-phosphotyrosine immunoprecipitates from ephrinB1-stimulated and unstimulated NG108-EphB2 cells.

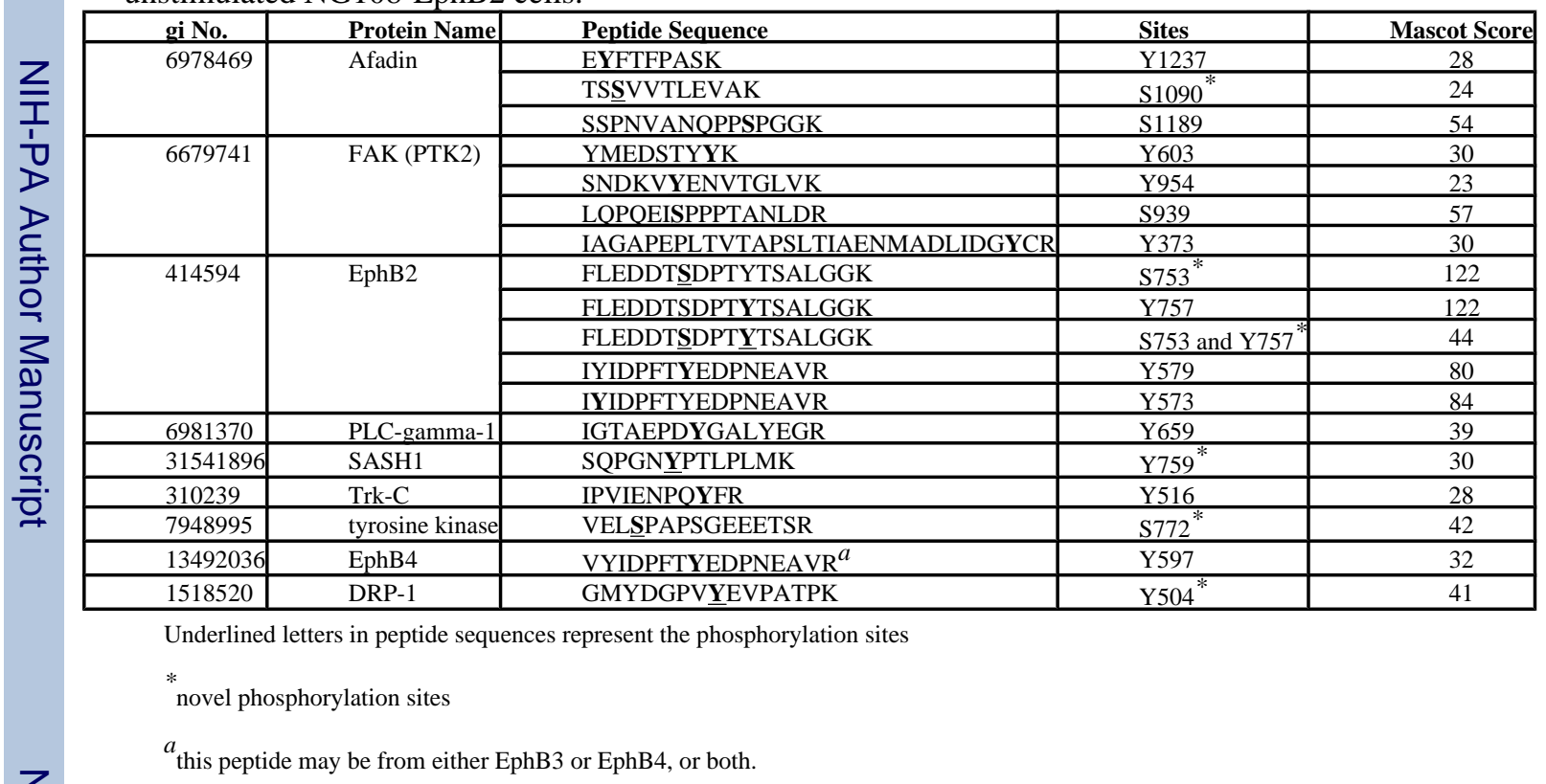

